(1)

CrossMark

\title{
Impact of tumour thickness on survival after radical radiation and surgery in malignant pleural mesothelioma
}

\author{
Marc de Perrot ${ }^{1}$, Zhi Dong ${ }^{2}$, Penelope Bradbury ${ }^{3}$, Demetris Patsios ${ }^{3}$, \\ Shaf Keshavjee', Natasha B. Leighl ${ }^{3}$, Andrew Hope ${ }^{4}$, Ronald Feld ${ }^{3}$ and John Cho ${ }^{4}$ \\ Affiliations: 'Division of Thoracic Surgery, Princess Margaret Cancer Centre and Toronto General Hospital, \\ University Health Network, University of Toronto, Toronto, ON, Canada. ${ }^{2}$ Dept of Medical Imaging. Princess \\ Margaret Cancer Centre and Toronto General Hospital, University Health Network, University of Toronto, \\ Toronto, ON, Canada. ${ }^{3}$ Division of Medical Oncology, Princess Margaret Cancer Centre and Toronto General \\ Hospital, University Health Network, University of Toronto, Toronto, ON, Canada. ${ }^{4}$ Dept of Radiation Oncology, \\ Princess Margaret Cancer Centre and Toronto General Hospital, University Health Network, University of \\ Toronto, Toronto, ON, Canada.
}

Correspondence: Marc de Perrot, Toronto General Hospital, 9N-961, 200 Elizabeth Street, Toronto, 0N M5G 2C4, Canada. E-mail: marc.deperrotQuhn.ca

@ERSpublications

Tumour thickness is an independent predictor of outcome after radical treatment for malignant pleural mesothelioma http://ow.ly/KCbT306wDBN

Cite this article as: de Perrot M, Dong Z, Bradbury P, et al. Impact of tumour thickness on survival after radical radiation and surgery in malignant pleural mesothelioma. Eur Respir J 2017; 49: 1601428 [https:// doi.org/10.1183/13993003.01428-2016].

ABSTRACT Tumour thickness was assessed to determine if this parameter could refine patients' selection for multimodality therapy in malignant pleural mesothelioma.

We reviewed 65 consecutive treatment-naïve malignant pleural mesothelioma patients undergoing surgery for mesothelioma after radiation therapy (SMART). Total tumour thickness was determined by measuring the maximal thickness on nine predefined sectors on the chest wall, mediastinum and diaphragm.

After a median follow-up of 19 months, 40 patients (62\%) developed recurrence and 36 died (55\%). Total tumour thickness, ranging between 2.4 and $21 \mathrm{~cm}$ (median $6.9 \mathrm{~cm}$ ), correlated with tumour volume $\left(\mathrm{p}<0.0001, \mathrm{R}^{2}=0.29\right)$ and maximum standardised uptake value $\left(\mathrm{p}=0.006, \mathrm{R}^{2}=0.11\right)$. Total tumour thickness had a significant impact on overall survival and disease-free survival in univariate analysis. In multivariate analysis, total tumour thickness remained an independent predictor of survival $(\mathrm{p}=0.02$, hazard ratio (HR) 1.12 , 95\% CI 1.02-1.23) and disease-free survival ( $\mathrm{p}=0.01$, HR 1.13,95\% CI 1.03-1.24) along with epithelial histologic subtype $(\mathrm{p}<0.0001, \mathrm{HR} 0.25,95 \%$ CI $0.13-0.50)$ and $\mathrm{pN} 2$ disease $(\mathrm{p}=0.03, \mathrm{HR} 2.15$, 95\% CI 1.07-4.33). Diaphragmatic tumour thickness correlated best with time to recurrence $(\mathrm{p}=0.002$, $\left.\mathrm{R}^{2}=0.22\right)$ and time to death $\left(\mathrm{p}=0.003, \mathrm{R}^{2}=0.2\right)$.

The impact of tumour thickness on survival and disease-free survival independent of histologic subtypes and nodal disease is extremely encouraging. This parameter could potentially be used to refine the clinical staging of malignant pleural mesothelioma and optimise patient selection for radical treatment.

Earn CME accreditation by answering questions about this article. You will find these at erj.ersjournals.com/journal/cme

This article has supplementary material available from erj.ersjournals.com

Received: July 162016 | Accepted after revision: Nov 132016

This study is registered as a clinical trial at ClinicalTrials.gov with identifier: NCT00797719.

Support statement: The authors would like to thank the Princess Margaret Hospital Foundation for supporting the clinical trial. Funding information for this article has been deposited with the Open Funder Registry.

Conflict of interest: Disclosures can be found alongside this article at erj.ersjournals.com

Copyright @ERS 2017 


\section{Introduction}

Malignant pleural mesothelioma (MPM) is an aggressive malignancy associated with poor outcome [1]. The role of surgery in the multimodal approach to this disease has remained controversial [2]. Part of the problem has been the poor clinical staging system, which limits our ability to adequately select patients for more aggressive approaches and prevents optimal comparisons between surgical and non-surgical series [3, 4].

In Toronto, we have developed an innovative approach with surgery for mesothelioma after radiation therapy (SMART). This approach consists of a short course of high-dose hemithoracic radiation followed by an extrapleural pneumonectomy (EPP) [5]. This approach has been shown to be feasible without increased morbidity compared to our previous experience with induction chemotherapy and has been associated with encouraging results in patients with epithelial MPM $[6,7]$.

The difficulty has been selecting appropriate patients for this approach. Patients with biphasic disease on diagnostic biopsy or with pathological N2 ( pN2) disease on endobronchial ultrasound (EBUS) have been excluded from this protocol. However, owing to limitations in accurate pre-operative staging, the final pathology is often discordant with the initial clinical impression, and we have seen a persistently high prevalence of $\mathrm{pN} 2$ disease and biphasic subtypes on the final surgical pathological specimen. The primary aim of this study was therefore to determine whether tumour thickness (TT) could be used to refine our selection criteria for the SMART approach.

Tumour volume, TT and maximum standardised uptake value (SUVmax) on ${ }^{18} \mathrm{~F}$-2-fluoro-2-deoxyD-glucose (FDG)-positron emission tomography-computed tomography (PET-CT) have been reported to correlate with outcome in MPM [8-11]. These criteria were therefore reviewed in our series of patients to determine their impact on outcome. TT was analysed in more detail owing to the potential ease of use of this marker in clinical practice.

\section{Methods}

\section{Patients}

All patients included in the SMART trial were reviewed. Patients undergoing chemotherapy before SMART were excluded from this analysis $(n=4)$. Patients selected for the SMART approach represented approximately $24 \%$ of all patients with mesothelioma seen in our centre [7]. The clinical stage was determined by CT scan of the chest and abdomen, PET-CT, and brain magnetic resonance imaging or CT. Pre-operative nodal sampling with EBUS has been routinely performed in our centre since December 2013. Patients with a diagnosis of biphasic MPM were excluded from the study after we completed an initial feasibility study with 25 patients [5]. The study was approved by our institutional review board.

\section{SMART protocol}

The SMART protocol includes a total of $25 \mathrm{~Gy}$ of radiation to the entire ipsilateral hemithorax by intensity-modulated radiation therapy, with a concomitant boost of $5 \mathrm{~Gy}$ to any gross tumour visible on CT or PET scan. Biopsy tract sites are included in the boost volumes. EPP is performed within a week after the end of radiation therapy [7]. Histological diagnosis and pathological staging is based on the 2004 World Health Organization (WHO) classification system and the TNM Classification of Malignant Tumours, 7th Edition, staging system [12]. Adjuvant chemotherapy with cisplatin and an antifolate (pemetrexed or raltitrexed) doublet are administered within 24 weeks after EPP for at least three cycles selectively to patients with pN2 disease on final pathological examination. After completing therapy, patients are followed at least every 3 months up to 2 years and then every 6 months up to 5 years. CT of the thorax and abdomen are performed at $3,6,12,18,24,36,48$ and 60 months or more frequently if clinically indicated. Additional tests are performed at the discretion of the investigators. Recurrences are treated off protocol.

\section{Radiation technique and tumour volume measurement}

The radiation field was defined by the clinical target volume targeting the entire ipsilateral hemithorax, from the thoracic inlet down to the diaphragmatic insertion. The gross tumour volume targeted any visible tumour on CT. FDG-PET was overlapped with the CT to ensure that all visible disease was encompassed. Gross tumour volume was then used to determine the tumour volume used in this analysis with the software Pinnacle version 8 (Philips Radiation Oncology Systems, Milpitas, CA, USA).

\section{Measurement of maximal tumour thickness}

All patients with digitally available axial, coronal and sagittal CT imaging with section thickness of $\leqslant 3 \mathrm{~mm}$ were included in the analysis. Five patients were excluded owing to the absence of adequate CT reconstruction images. TT was measured in a blinded fashion at nine separate sites on the chest wall, mediastinum and diaphragm according to the modified RECIST criteria (supplementary figure S1). The chest wall was divided into three sectors on axial CT: anterior third, middle third and posterior third. The mediastinum was divided 
into three sectors on axial CT: upper mediastinum, corresponding to the mediastinum above the mainstem bronchus; lower anterior mediastinum, corresponding to the mediastinum lower and anterior to the mainstem bronchus; and lower posterior mediastinum, corresponding to the mediastinum lower and posterior to the mainstem bronchus. The diaphragm was divided into three sectors on the sagittal reconstructions: anterior third, middle third and posterior third. Total TT was defined by the sum of all nine TTs measured on the chest wall, mediastinum and diaphragm. The chest wall TT was defined by the sum of the three chest wall sectors. The mediastinal TT was defined by the sum of the three mediastinal sectors. The diaphragmatic TT was defined by the sum of the three diaphragmatic sectors.

\section{Statistical analysis}

Demographic and treatment data are reported as mean \pm SD or median (range). All patients were followed up to June 2016 or until death to have at least 6 months of follow-up for all patients alive. All but one patient were followed with serial CT as planned. One patient who died from recurrence had no follow-up CT beyond 1 year and was removed from the disease-free survival analysis. Survival was calculated from the starting date of radiation therapy using the Kaplan-Meier method. Overall survival was calculated up to the date of death or censored at the date of last follow-up for patients still alive. Disease-free survival was calculated up to the date of a recurrence of MPM or censored at the date of last follow-up or death in the absence of relapse. Differences in survival were tested for significance using the log-rank test. Univariate and multivariate Cox regression analysis was used to determine factors associated with survival. Correlations were evaluated by linear regression analysis. For comparison in survival, the Zurich score and the European Organisation for Research and Treatment of Cancer (EORTC) score were calculated for all patients. The Zurich score was calculated with two variables, namely tumour volume $>500 \mathrm{~cm}^{3}$ pre-radiation and non-epithelial histology pre-radiation [8]. C-reactive protein levels were not available and were therefore excluded from the scoring system. The EORTC score was calculated based on the predefined formula [13, 14]. Statview V (Abacus Concept, Berkeley, CA, USA) was used for all analyses. A p-value $<0.05$ was considered significant.

\section{Results}

A total of 65 consecutive treatment-naive MPM patients undergoing the SMART approach between November 2008 and October 2015 were included in this study. The vast majority of patients were male (83\%), ranging in age between 41 and 82 years (median 64 years old). Staging EBUS was performed in 19 patients. Median tumour volume was $687 \mathrm{~cm}^{3}$ and median SUVmax was 5 (table 1). Chest wall TT ranged between 0.6 and $7.9 \mathrm{~cm}$ (median $2.1 \mathrm{~cm}$ ), mediastinal TT ranged between 0.3 and $6.7 \mathrm{~cm}$ (median $1.7 \mathrm{~cm}$ ), and diaphragmatic TT ranged between 0.9 and $6.7 \mathrm{~cm}$ (median $2.4 \mathrm{~cm}$ ). Total TT ranged between 2.4 and $21 \mathrm{~cm}$ (median $6.9 \mathrm{~cm}$ ). Total TT correlated with tumour volume and SUVmax (figure 1). Total TT did not correlate with the percentage of perfusion to the diseased lung $\left(\mathrm{p}=0.7, \mathrm{R}^{2}=0.01\right)$.

All patients completed the hemithoracic radiation and EPP as planned. There was no 30-day or hospital mortality. All but five patients were staged $3(n=25)$ or $4(n=35)$ on final pathology. Final histology of the tumours was epithelial in 46 patients (71\%) and biphasic in 19 (29\%). A total of 34 patients (52\%) were diagnosed with pN2 disease, of whom 12 (18\% of the whole cohort) underwent three or four cycles of adjuvant chemotherapy. Three patients died without any evidence of tumour recurrence. Two of them died within 3 months after hospital discharge (90-day mortality of 3\%) from, respectively, a cardiorespiratory arrest of unknown cause and an empyema. A third patient died 38 months after SMART from septic complications post-cholecystectomy without any clinical or radiological evidence of mesothelioma recurrence.

$\begin{array}{lc}\text { TABLE } 1 \text { Patients' characteristics } & \\ \text { Subjects } \mathrm{n} & 65 \\ \text { Age years } & 64(41-82) \\ \text { Sex } \mathrm{n}(\mathrm{M} / \mathrm{F}) & 54 / 11 \\ \text { Side of disease } \mathrm{n}^{(\text {right/left) }} & 48 / 17 \\ \text { Tumour volume } \mathrm{cm}^{3} & 687(0-3539) \\ \text { SUVmax } & 5(1.2-17.2) \\ \text { Diaphragmatic TT cm } & 2.4(0.9-6.7) \\ \text { Chest wall TT cm } & 2.1(0.6-7.9) \\ \text { Mediastinal TT cm } & 1.7(0.3-6.7) \\ \text { Total TT cm } & 6.9(2.4-21)\end{array}$

Data are presented as median (range), unless otherwise indicated. SUVmax: maximum standardised uptake value; TT: tumour thickness. 

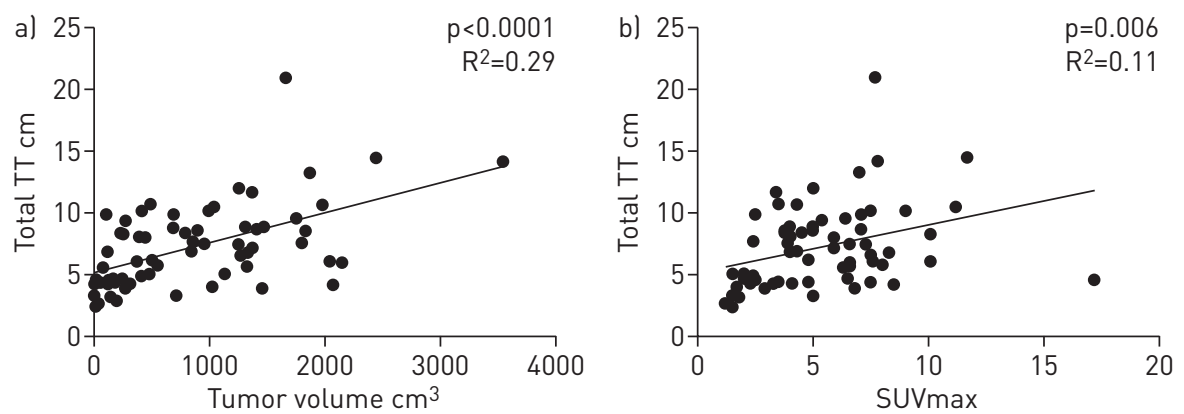

FIGURE 1 Total tumour thickness (TT) correlated with a) tumour volume and b) maximum standardised uptake value (SUVmax).

After a median follow-up of 19 months, a total of 40 patients (62\%) developed recurrence, of whom 36 died (55\%). As previously reported [5, 7], a biphasic histologic subtype had a negative impact on disease-free survival (median disease-free survival of 8 months versus 30 months in epithelial subtypes, $\mathrm{p}<0.0001$ ) and overall survival (median survival of 14 months versus 36 months in epithelial subtypes, $\mathrm{p}=0.0006$ ). The presence of $\mathrm{pN} 2$ disease also had a negative impact on disease-free survival (median disease-free survival of 10 months versus 29 months in $\mathrm{pN} 0-1, \mathrm{p}=0.01$ ) and overall survival (median survival of 16 months versus 36 months in $\mathrm{pN} 0-1, \mathrm{p}=0.02$ ). In contrast, the pathological tumour ( $\mathrm{pT}$ ) status had no impact on disease-free survival (median disease-free survival of 16 months in pT4 versus 19 months in $\mathrm{pT} 1-3, \mathrm{p}=0.5$ ) and overall survival (median survival of 24 months in pT4 versus 29 months in pT1-3, p=0.9).

Among the pre-operative variables tested in univariate analysis, total TT and tumour volume had a significant impact on overall survival, whereas sex, age and side of disease had no impact on outcome (table 2). Tumour volume $(\mathrm{p}=0.03)$ and total TT $(\mathrm{p}<0.0001)$ also had a significant impact on disease-free survival. SUVmax had a limited impact on overall survival $(\mathrm{p}=0.05)$ and did not have any impact on disease-free survival $(\mathrm{p}=0.2)$.

Survival analysis based on the Zurich score demonstrated good stratification in overall survival and disease-free survival (figure 2). A median survival of 51 months was achieved in patients with a tumour volume $<500 \mathrm{~cm}^{3}$ and epithelial histology on the initial biopsy, while the median survival was 18 months in patients with a Zurich score of 1 and 12 months in patients with a score of 2 . The EORTC score did not show any significant difference in survival, with a median survival of 28 months when the score was $<1.27$ and 27 months when the score was $>1.27(\mathrm{p}=0.9)$.

After separating total TT into two groups according to the median measurement, patients with total TT $<7 \mathrm{~cm}$ reached a median survival of 45 months and disease-free survival of 32 months, while those with total TT $\geqslant 7 \mathrm{~cm}$ had a median survival of 18 months and a median disease-free survival of 11 months (figure 3). In a multivariate analysis assessing pre- and post-operative variables, total TT remained a significant predictor of overall survival and disease-free survival along with histologic subtype (table 3 ). The presence of $\mathrm{pN} 2$ disease was a significant predictor of disease-free survival, but not overall survival.

Although the coefficient of determination $\left(\mathrm{R}^{2}\right)$ was low, total TT correlated with time to recurrence and time to death (figure 4). A specific analysis of chest wall, mediastinal and diaphragmatic TT demonstrated that the best correlation was provided by the diaphragmatic TT. Delayed recurrence beyond 3 years was seen predominantly in diaphragmatic $\mathrm{TT}<2 \mathrm{~cm}$, while early recurrence within 18 months was seen

TABLE 2 Impact of pre-operative variables on overall survival

\begin{tabular}{lccc} 
Variables & \multicolumn{3}{c}{ Unvariate analysis } \\
\cline { 2 - 4 } & p-value & HR & $\mathbf{9 5 \%} \mathbf{C l}$ \\
\hline Sex (male) & 0.4 & 1.39 & $0.61-3.16$ \\
Age (continuous) & 0.3 & 1.02 & $0.98-1.062$ \\
Side of disease (right) & 0.6 & 0.84 & $0.41-1.73$ \\
SUVmax (continuous) & 0.05 & 1.09 & $0.99-1.19$ \\
Tumour volume (continuous) & 0.004 & 1.00 & $1.00-1.001$ \\
Total TT (continuous) & 0.0002 & 1.15 & $1.07-1.24$ \\
\hline
\end{tabular}

HR: hazard ratio; SUVmax: maximum standardised uptake value; TT: tumour thickness. 

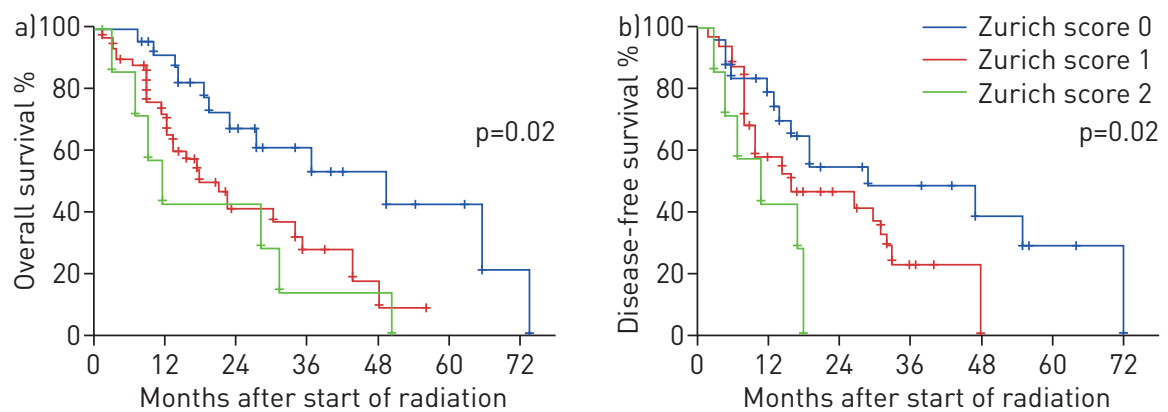

FIGURE 2 a) Overall survival and b) disease-free survival according to the prognostic score developed in Zurich based on the presence of tumour volume $>500 \mathrm{~cm}^{3}$ or non-epithelial histology before starting treatment.
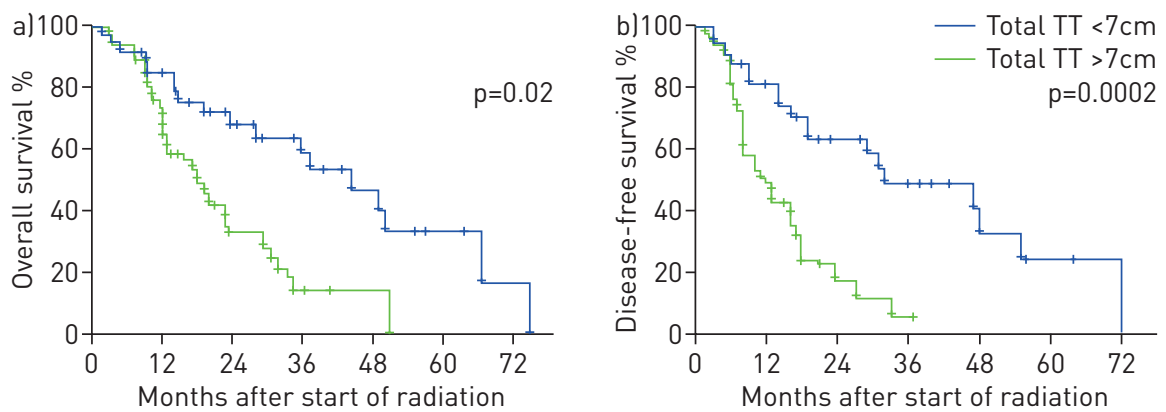

FIGURE 3 a) Overall survival and b) disease-free survival according to total tumour thickness (TT) smaller or greater than $7 \mathrm{~cm}$.

predominantly when the diaphragmatic TT was $>4 \mathrm{~cm}$. Survival breakdown according to TT cut-offs showed that patients with diaphragmatic TT $<2 \mathrm{~cm} \quad(n=21)$ had a median disease-free survival of 55 months compared to 14 months in patients with diaphragmatic TT 2-3.9 cm (n=33) and 10 months in patients with diaphragmatic TT $\geqslant 4 \mathrm{~cm}(\mathrm{n}=11)$ (figure 5).

Recurrence was demonstrated in 40 patients (62\%), 18 of whom had more than one site of recurrence. Sites of recurrence included the contralateral chest $(n=23)$, abdomen $(n=17)$, ipsilateral chest $(n=11)$, mediastinal nodes $(n=5)$ and pericardium $(n=2)$. Patients with abdominal recurrence had greater diaphragmatic TT than other patients $(3.3 \pm 1.6 \mathrm{~cm}$ versus $2.5 \pm 1.1 \mathrm{~cm}$, respectively; $\mathrm{p}=0.03)$. The mediastinal TT and chest wall TT had no significant impact on the risk of abdominal, contralateral chest or ipsilateral chest recurrence.

\section{Discussion}

This study looking at clinical parameters of outcome in a consecutive group of patients treated with pre-operative accelerated hemithoracic radiation followed by EPP demonstrates that TT could be an excellent predictor of survival. The impact of this variable on survival independent of histology and nodal disease is extremely encouraging because, for the first time, an easily measurable radiological parameter

\section{TABLE 3 Multivariate Cox regression analyses predicting survival after SMART}

\section{Survival}

Variables

-value

Epithelial histologic subtype

Presence of pN2 disease

Disease-free survival

Total TT (continuous)

Epithelial histologic subtype

Presence of pN2 disease
0.02

0.005

0.3

$\begin{array}{cr}0.01 & 1.13 \\ <0.0001 & 0.25 \\ 0.03 & 2.15\end{array}$

1.12

0.39

1.49

$0.21-0.76$

$0.73-3.07$

$1.03-1.24$

$0.13-0.5$

$1.07-4.33$

HR: hazard ratio; TT: tumour thickness. 

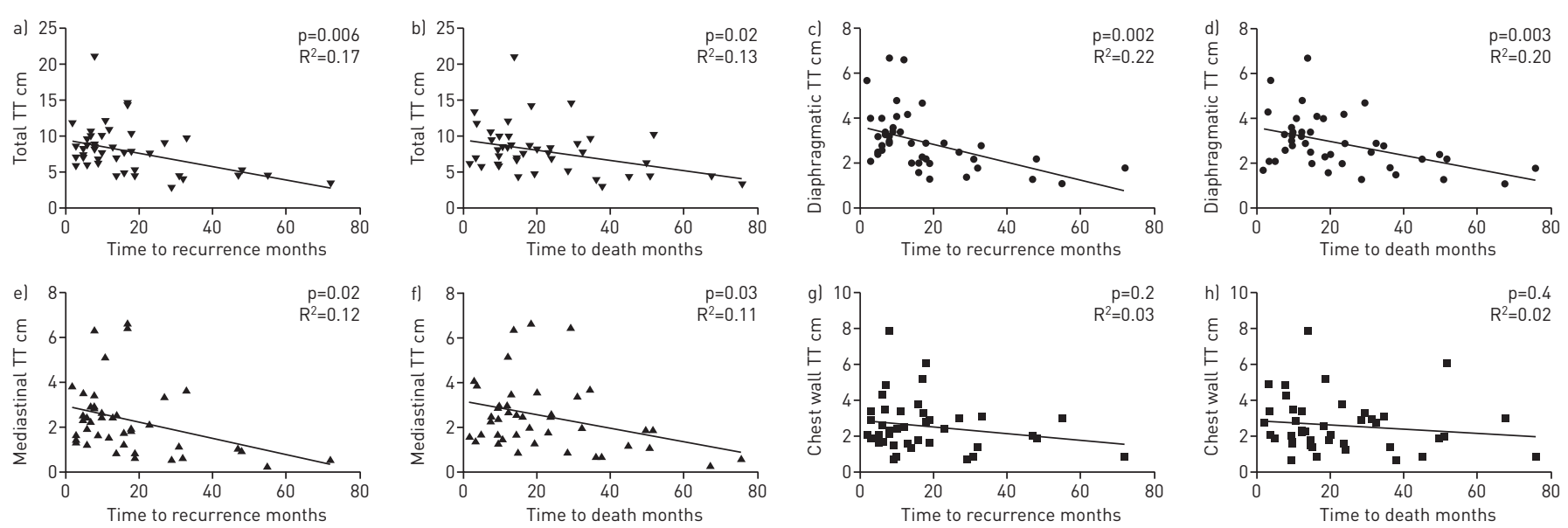

FIGURE 4 a, b) Total tumour thickness (TT), c, d) diaphragmatic TT and e, f) mediastinal TT correlated with time to recurrence and time to death. $g, h)$ There was no correlation with time to recurrence and time to death in chest wall TT. Patients without recurrence were excluded from this analysis.

could potentially be used to refine the clinical staging of MPM. The diaphragmatic TT provided the best correlation with survival and could potentially be an important parameter to determine the clinical stage.

The SMART approach provides encouraging results in patients with epithelial subtypes compared to outcomes reported for this patient group in other large multimodal prospective clinical trials [8, 15-17]. Direct comparison between studies is, however, limited by the lack of adequate clinical staging potentially affecting patients' selection. Hence, if TT is confirmed to impact survival in other surgical and non-surgical series, this parameter could help to refine the clinical staging, allow more appropriate comparison between clinical trials, and optimise patients' selection for an aggressive approach.

As reported previously, we confirm that tumour volume is a potential marker of outcome and that the scoring system developed in Zurich based on the pre-treatment histologic subtype and tumour volume provides a good stratification of survival $[8,9,18]$. However, the benefit of tumour volume is currently limited by the lack of standardisation of the software used and the inability to do this evaluation easily in the clinic when patients come for assessment. In this study, we used the gross tumour volume from the radiation boost to define the tumour volume. Because the radiation boost targets all visible disease seen on the CT and PET scan, this value provided a good estimate of the tumour volume. Our tumour volumes were of a similar range to those reported in other series, with a median tumour volume of $687 \mathrm{~cm}^{3}$ and a maximal tumour volume of $3539 \mathrm{~cm}^{3}[8,9]$. As previously reported, we observed that the group of patients with a tumour volume of $<500 \mathrm{~cm}^{3}$ and an epithelial subtype had better outcomes, with a median survival reaching 51 months $[9,18]$.

TT is routinely used to assess tumour response in MPM patients as part of the modified RECIST criteria; a recent analysis from the International Association for the Study of Lung Cancer (IASLC) database demonstrated the potential impact of TT on survival in a large cohort of patients with MPM [19, 20].
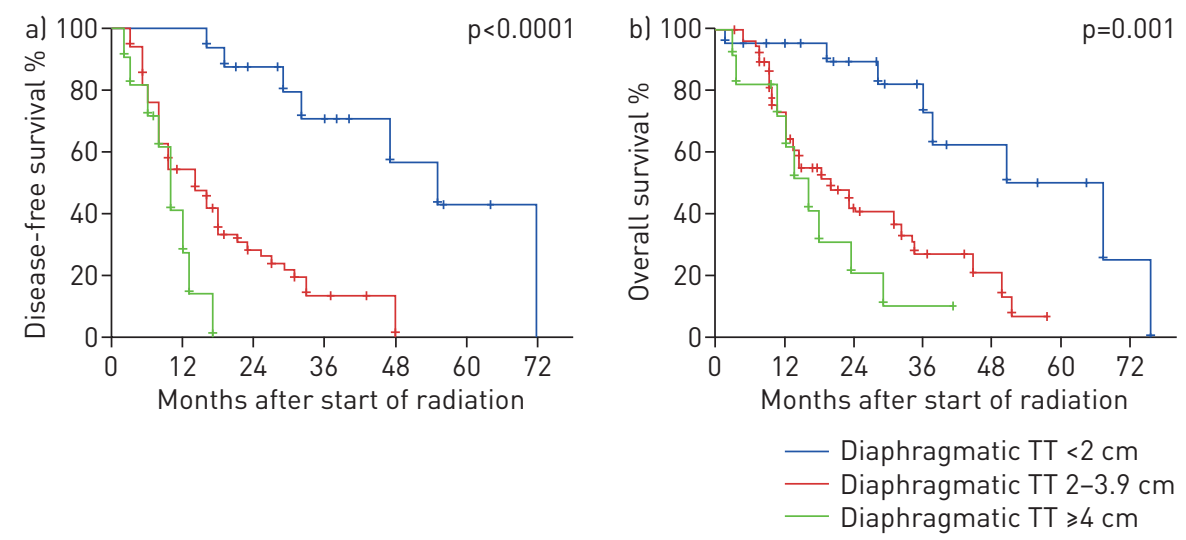

FIGURE 5 a) Disease-free survival and b) overall survival based on diaphragmatic tumour thickness (TT). 
Over the past two decades, improvements in CT technology with slices of $\leqslant 3 \mathrm{~mm}$ have led to an acceptable measurement of TT down to $7.5 \mathrm{~mm}$ in patients with MPM [21]. Another major improvement in CT technology over the recent years has been the routine use of coronal and sagittal reconstructions with thin slices. A particular benefit of these reconstructions has been the quality of assessment of the diaphragm $[22,23]$. However, no study has yet assessed the role of these CT reconstruction images in the evaluation of TT on the diaphragm. Considering the impact of diaphragmatic TT in this study, it would be worthwhile to analyse this parameter in a larger cohort of patients to determine whether it provides additional benefits over chest wall or mediastinal TT.

This study is limited by the small number of patients. All patients were also uniformly treated with the same approach, which provides some benefit when studying a relatively small group of patients, but limits the generalisation of our findings. Hence, further studies will have to be performed to confirm if TT remains a predictor of outcome with other types of therapeutic approach, particularly in non-surgical groups.

In conclusion, this study demonstrates the potential impact of TT on survival. This variable could provide a reliable and easily measurable parameter to refine clinical staging. Further studies will be required to confirm this encouraging finding.

\section{References}

1 Baas P, Fennell D, Kerr KM, et al. Malignant pleural mesothelioma: ESMO Clinical Practice Guidelines for diagnosis, treatment and follow-up. Ann Oncol 2015; 26: Suppl. 5, v31-v39.

2 Van Schil PE, Opitz I, Weder W, et al. Multimodal management of malignant pleural mesothelioma: where are we today? Eur Respir J 2014; 44: 754-764.

3 Rusch VW, Giroux D. Do we need a revised staging system for malignant pleural mesothelioma? Analysis of the IASLC database. Ann Cardiothorac Surg 2012; 1: 438-448.

4 Frauenfelder T, Kestenholz P, Hunziker R, et al. Use of computed tomography and positron emission tomography/computed tomography for staging of local extent in patients with malignant pleural mesothelioma. J Comput Assist Tomogr 2015; 39: 160-165.

5 Cho BC, Feld R, Leighl N, et al. A feasibility study evaluating Surgery for Mesothelioma After Radiation Therapy: the "SMART" approach for resectable malignant pleural mesothelioma. J Thorac Oncol 2014; 9: 397-402.

6 Mordant $\mathrm{P}$, McRae K, Cho J, et al. Impact of induction therapy on postoperative outcome after extrapleural pneumonectomy for malignant pleural mesothelioma: does induction-accelerated hemithoracic radiation increase the surgical risk? Eur J Cardiothorac Surg 2016; 50: 433-438.

7 de Perrot $M$, Feld R, Leighl NB, et al. Accelerated hemithoracic radiation followed by extrapleural pneumonectomy for malignant pleural mesothelioma. J Thorac Cardiovasc Surg 2016; 151: 468-475.

8 Opitz I, Friess M, Kestenholz P, et al. A New prognostic score supporting treatment allocation for multimodality therapy for malignant pleural mesothelioma: a review of 12 years' experience. J Thorac Oncol 2015; 10: 1634-1641.

9 Gill RR, Richards WG, Yeap BY, et al. Epithelial malignant pleural mesothelioma after extrapleural pneumonectomy: stratification of survival with CT-derived tumor volume. AJR Am J Roentgenol 2012; 198: 359-363.

10 Klabatsa A, Chicklore S, Barrington SF, et al. The association of 18F-FDG PET/CT parameters with survival in malignant pleural mesothelioma. Eur J Nucl Med Mol Imaging 2014; 41: 276-282.

11 Flores RM. The role of PET in the surgical management of malignant pleural mesothelioma. Lung Cancer 2005; 49: Suppl. 1, S27-S32.

12 Travis WD, Brambilla E, Muller-Hermelink KM. Pathology and Genetics: Tumours of the Lung, Pleura, Thymus and Heart. Lyon, IARC, 2004.

13 Curran D, Sahmoud T, Therasse P, et al. Prognostic factors in patients with pleural mesothelioma: the European Organization for Research and Treatment of Cancer experience. J Clin Oncol 1998; 16: 145-152.

14 Fennell DA, Parmar A, Shamash J, et al. Statistical validation of the EORTC prognostic model for malignant pleural mesothelioma based on three consecutive phase II trials. J Clin Oncol 2005; 23: 184-189.

15 Weder W, Stahel RA, Bernhard J, et al. Multicenter trial of neo-adjuvant chemotherapy followed by extrapleural pneumonectomy in malignant pleural mesothelioma. Ann Oncol 2007; 18: 1196-1202.

16 Van Schil PE, Baas P, Gaafar R, et al. Trimodality therapy for malignant pleural mesothelioma: results from an EORTC phase II multicentre trial. Eur Respir J 2010; 36: 1362-1369.

17 Krug LM, Pass HI, Rusch VW, et al. Multicenter phase II trial of neoadjuvant pemetrexed plus cisplatin followed by extrapleural pneumonectomy and radiation for malignant pleural mesothelioma. J Clin Oncol 2009; 27: 3007-3013.

18 Pass HI, Temeck BK, Kranda K, et al. Preoperative tumor volume is associated with outcome in malignant pleural mesothelioma. J Thorac Cardiovasc Surg 1998; 115: 310-317.

19 Byrne MJ, Nowak AK. Modified RECIST criteria for assessment of response in malignant pleural mesothelioma. Ann Oncol 2004; 15: 257-260.

20 Nowak AK, Chansky K, Rice DC, et al. The IASLC Mesothelioma Staging Project: Proposals for revisions of the $\mathrm{T}$ descriptors in the forthcoming eighth edition of the TNM classification for pleural mesothelioma. $J$ Thorac Oncol 2016; 11: 2089-2099.

21 Armato SG 3rd, Nowak AK, Francis RJ, et al. Observer variability in mesothelioma tumor thickness measurements: defining minimally measurable lesions. J Thorac Oncol 2014; 9: 1187-1194.

22 Sandrasegaran K, Rydberg J, Tann M, et al. Benefits of routine use of coronal and sagittal reformations in multi-slice CT examination of the abdomen and pelvis. Clin Radiol 2007; 62: 340-347.

23 Rydberg J, Sandrasegaran K, Tarver RD, et al. Routine isotropic computed tomography scanning of chest: value of coronal and sagittal reformations. Invest Radiol 2007; 42: 23-28. 